\title{
Simulations de grandes échelles : aspects mathématiques et numériques
}

\author{
Georges-Henri Cottet, Delia Jiroveanu et Bertrand Michaux
}

\begin{abstract}
Résumé. La simulation des écoulements turbulents ou à haut nombre de Reynolds ne peut pas en général être menée avec un nombre de degrés de liberté suffisant pour représenter toutes les échelles de l'écoulement. Les Simulations des Grandes Ėchelles (SGE) visent à ne simuler que les échelles significatives pour un cot de calcul raisonnable. Ces simulations s'appuient sur des modèles, dits modèles sous-maille, paramétrant l'effet des échelles non résolues sur les grandes échelles, notamment en dissipant l'énergie qui a tendance à s'accumuler en bout de spectre. Ces modèles sont en général déduits d'arguments physiques de type cascade d'énergie. D'un point de vue mathématique, les modèles SGE ont des liens étroits avec les techniques de déconvolution. Ils peuvent être aussi considérés comme des régularisations minimales des équations de Navier-Stokes possédant des solutions régulières. On décrira des travaux récents dans ces deux directions, ainsi que les algorithmes numériques qui s'en déduisent. On montrera en particulier comment apparaissent naturellement des modèles de diffusion anisotropes qui s'implantent facilement dans les codes classiques. On montera enfin comment les méthodes numériques lagrangiennes se distinguent des méthodes eulériennes dans le contexte des SGE.
\end{abstract}

Mots clés. Simulation grandes échelles, modèles sous-maille, écoulements turbulents.

\begin{abstract}
Computation of turbulent flows or of flows at high Reynolds numbers can not in general be done with a number of degrees of freedom sufficient to represent all the scales of the flow. The aim of Large Eddy Simulations (LES) is to compute only the relevant scales at a reasonable cost. These simulations are based on models (the so-called subgrid models) which report the effects of the small scales on the large ones, in particular by a dissipation of the energy which accumulates in the tail. They are usually derived from physical arguments based on energy cascade. From a mathematical standpoint, the LES models are closely linked to the deconvolution techniques. They can also be considered as minimal regularizations of the Navier-Stokes equations. with smooth solutions. Recent work in those two directions will be presented, as well as numerical algorithms. It will be shown how anisotropic diffusion models naturally appears and can easilly be implemented in classical codes. Finally emphasis will be put on the difference between Eulerian and Lagrangian methods in the SGE framework.
\end{abstract}

\section{Introduction}

Le but des simulations de grandes échelles est de ne calculer avec précision que les échelles significatives d'un écoulement. Pour cela on cherche à résoudre les équations de Navier-Stokes 
moyennées en espace sur une échelle $\Delta$, dite échelle de coupure, en général fonction de la résolution maximale que l'on peut s'offrir dans un schéma numérique donné.

La difficulté est évidemment que ces équations ne sont pas fermées puisque, si l'on note $\overline{\mathbf{u}}$ la moyenne locale d'un champ de vitesse on a $\bar{u}_{i} \bar{u}_{j} \neq \overline{u_{i} u_{j}}$. Autrement dit l'interaction non linéaire des grandes échelles peut produire des petites échelles. D’un autre coté les physiciens savent qu'une des caractéristiques fondamentales de la turbulence 3D est que l'énergie des grandes échelles est constamment "pompée " vers les plus petites échelles, jusqu'à atteindre des nombres d'onde suffisamment élevés pour que la viscosité moléculaire, aussi faible soit-elle, puisse la dissiper. Si l'on veut donc fermer les équations de Navier-Stokes moyennées, il faut y adjoindre des termes de dissipation, dite dissipation sous-maille, qui se substituent à ce mécanisme de cascade d'énergie [6].

Le terme de dissipation le plus simple et qui continue à servir de base aux modèles modernes, est du à Smagorinsky et s'écrit sous la forme $\operatorname{div}\left(\nu_{t} \nabla \mathbf{u}\right)$ avec $\nu_{t}=C \Delta^{2}|\nabla \mathbf{u}|$. Ce modèle est en fait très rudimentaire et beaucoup trop dissipatif, comme le montrent des comparaisons entre ses prédictions et les échanges d'energie sous-maille calculées par résolution directe des équations de Navier-Stokes sur des grilles très fines. Pour améliorer ses performances, on fait souvent appel à des modèles qui calculent de manière adaptative en temps et en espace le coefficient $C$ [5]. Sans rentrer dans les détails, disons que cette technique fonctionne très bien dans des géométries simples mais pose certains problèmes dans des cas plus généraux.

Adoptons maintenant un point de vue différent. Puisqu'ils sont censés modéliser des équations moyennées, les modèles sous-maille doivent assurer l'existence de solutions régulières pour tout temps. En fait cette propriété, qui reste un problème ouvert pour les équations de Navier-Stokes elles-mêmes, peut être assez facilement démontrée pour le modèle de Smagorinsky. L'excès de dissipation constaté pour ce modèle amène alors à se poser la question suivante: quel modèle de dissipation minimal doit-on ajouter aux équations de Navier-Stokes pour assurer l'existence de solutions régulières pour tout temps.

Dans la suite de l'exposé nous abordons cette question pour les écoulements incompressibles du point de vue de la formulation tourbillon des équations de Navier-Stokes, car c'est probablement celui qui met le mieux en évidence les possibilités de singularité en dimension 3 [1,7]. A partir de cette formulation, nous écrivons un modèle sous-maille faisant intervenir un tenseur de viscosité puis nous montrons, en revenant à la formulation vitesse-pression, comment ce modèle s'implante dans des codes de type différences finies. Nous discutons ensuite le rôle particulier des schémas Lagrangiens du point de vue de la Simulation des Grandes Echelles. Ces diverses questions sont illustrées par des résultats numériques soit en turbulence homogène soit sur des écoulements laminaires à haut nombre de Reynolds. 


\section{Formulation vitesse-tourbillon des équations de Navier-Stokes et tenseurs de viscosité turbulente}

Pour simplifier l'exposé on se placera dans le cas de conditions aux limites périodiques. La formulation vitesse-tourbillon des équations. de Navier-Stokes s'écrit

$$
\frac{\partial \boldsymbol{\omega}}{\partial t}+(\mathbf{u} \cdot \nabla \boldsymbol{\omega})-(\boldsymbol{\omega} \cdot \nabla) \mathbf{u}-\nu \Delta \boldsymbol{\omega}=0
$$

Le terme de déformation de tourbillon $(\boldsymbol{\omega} \cdot \nabla) \mathbf{u}$, nul en dimension 2, est responsable de la réorientation et de l'intensifaction local du tourbillon, deux caractéristiques essentielles des écoulements tri-dimensionnels. Si $\nu=0$, il agit comme un forcing quadratique sur la partie convection du tourbillon, susceptible de produire un excès d'enstrophie que la viscosité ne suffit pas nécessairement à contre-balancer. Si l'on cherche maintenant un terme de dissipation capable d'éviter l'apparition de singularités, une idée naturelle est de choisir l'intensité de cette diffusion proportionnelle au taux d'étirement du tourbillon. On obtient alors le terme de $\operatorname{dissipation} \operatorname{div}\left(\nu_{t} \nabla \boldsymbol{\omega}\right)$, avec $\nu_{t}=C \Delta^{2}|\nabla \mathbf{u}|$, qui est évidemment une variante tourbillon du modèle de Smagorinsky. Comme on l'a déjà dit ce modèle se révèle en pratique trop dissipatif. D'un autre côté on peut évidemment écrire

$$
\int(\boldsymbol{\omega} \cdot \nabla) \mathbf{u} \cdot \mathbf{u} d x=\int \omega_{i} S_{i j} \omega_{j} d x \leq \int \omega_{i} S_{i j}^{+} \omega_{j} d x
$$

où $S_{i j}=1 / 2\left(\partial u_{i} / \partial x_{j}+\partial u_{j} / \partial x_{i}\right)$ dénote le tenseur (symétrique) de déformation et $S_{i j}^{+}$sa partie positive. Un nouveau choix de dissipation sous-maille consiste alors a prendre

$$
\nu_{t}=C \Delta^{2}\left[S_{i j}^{+}\right]
$$

Il existe évidemment d'autres choix naturels. Tout tenseur ayant la même "intensité" que $S^{+}$peut convenir. Comme dans un écoulement incompressible la somme de toutes les valeurs propres de $S$ est nulle, on peut par exemple considérer le tenseur $-S^{-}$. La différence entre ces modèles tient aux directions de dissipation qui leur sont associées. Pour faire le bon choix il est utile de revenir à la motivation physique du modèle recherché, à savoir dissiper les petites échelles produites dans l'écoulement. Le schéma de la figure 2 permet de comprendre ce qui se passe au voisinage d'un point hyperbolique, avec 2 valeurs propres de signe opposé, en dimension 2 pour simplifier. Il est clair que les petites échelles sont produites dans les directions propres associées aux valeurs propres négatives de $S$. C'est donc dans ces directions qu'il faut dissiper de l'enstrophie, ce qui conduit au choix

$$
\nu_{t}=-C \Delta^{2} S^{-}
$$

Le modèle final est obtenu en ajoutant un terme de pression de manière à assurer que la vorticité reste à divergence nulle:

$$
\frac{\partial \boldsymbol{\omega}}{\partial t}+(\mathbf{u} \cdot \nabla \boldsymbol{\omega})-(\boldsymbol{\omega} \cdot \nabla) \mathbf{u}-\nu \Delta \boldsymbol{\omega}+C \Delta^{2} \operatorname{div}\left(S^{-} \nabla \boldsymbol{\omega}\right)+\nabla q=0
$$

Revenant maintenant à la formulation vitesse-pression on obtient finalement le modèle

$$
\frac{\partial \mathbf{u}}{\partial t}+(\mathbf{u} \cdot \nabla \mathbf{u})-\nu \Delta \mathbf{u}+C \Delta^{2} \operatorname{div}\left(S^{-} \nabla \mathbf{u}\right)+\nabla p=0
$$




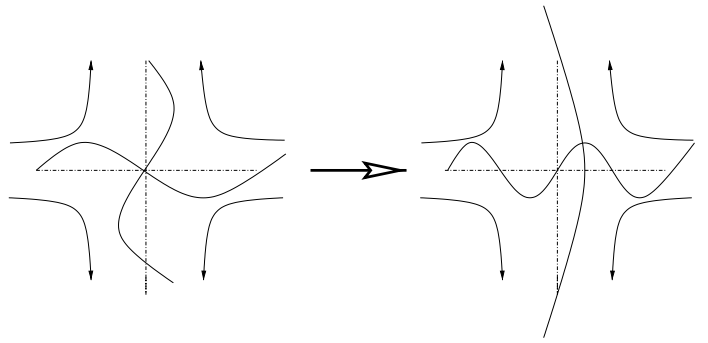

Figure 1: Ecoulement autour d'un point hyperbolique et incidence sur les échelles convectées

\section{Discrétisation dans un schéma Eulérien}

La mise en oeuvre de (2) ou (3) dans un schéma Eulérien - disons différences finies pour simplifier - semble à première vue délicate car elle suppose la diagonalisation d'un tenseur $3 \times 3$ en chaque point de grille, ce qui peut s'avérer coûteux.

On peut s'affranchir de ce calcul en commençant par approcher le terme $\operatorname{div}(S \nabla u)$ par une intégrale puis en faisant un bilan d'énergie local sur les contributions des points de quadrature.

Plus précisément, des développements de Taylor standard (voir [4]) permettent d'écrire

$$
\operatorname{div}(S \nabla u) \simeq-\Delta^{-6} \int\left[u_{j}(\mathbf{y})-u_{j}(\mathbf{x})\right]\left[u_{i}(\mathbf{y})-u_{i}(\mathbf{x})\right] \partial_{j} \zeta\left(\frac{\mathbf{y}-\mathbf{x}}{\Delta}\right) d \mathbf{y}
$$

où $\zeta$ est une fonction cut-off vérifiant

$$
\int x_{k} x_{l} \zeta(\mathbf{x}) d \mathbf{x}=\delta_{k l}
$$

Si maintenant on multiplie scalairement par $\mathbf{u}$ l'équation (4), les propiétés de symmétrie de $\zeta$ permettent d'écrire

$$
\int S \nabla \mathbf{u} \cdot \nabla \mathbf{u}=\frac{1}{2} \Delta^{-6} \int[\mathbf{u}(\mathbf{y})-\mathbf{u}(\mathbf{x})] \cdot \nabla \zeta\left(\frac{\mathbf{y}-\mathbf{x}}{\Delta}\right)|\mathbf{u}(\mathbf{y})-\mathbf{u}(\mathbf{x})|^{2} d \mathbf{x} d \mathbf{y}
$$

La contribution dissipative du tenseur $S$ peut donc être obtenue en ne gardant que les points d'intégration vérifiant

$$
[\mathbf{u}(\mathbf{y})-\mathbf{u}(\mathbf{x})] \cdot \nabla \zeta\left(\frac{\mathbf{y}-\mathbf{x}}{\Delta}\right) \geq 0
$$

Le terme de dissipation sous-maille s'écrit finalement

$$
\left.C \Delta^{-4} \int\left\{[\mathbf{u}(\mathbf{x})-\mathbf{u}(\mathbf{y})] \cdot \nabla \zeta\left(\frac{\mathbf{x}-\mathbf{y}}{\Delta}\right)\right]\right\}_{+}[\mathbf{u}(\mathbf{x})-\mathbf{u}(\mathbf{y})] d \mathbf{y}
$$

où $a_{+}=\max (0, a)$. En pratique on choisit un cut-off positif à symmétrie sphérique, et on discrétise l'intégrale ci-dessus sur les points de grille les plus proches. Dans le cas d'une grille cartésienne uniforme et un cut-off linéaire par morceaux on obtient un schéma de différences finies symbolisé par la figure 3. On voit que, dans le cas de l'écoulement schématisé dans la figure 2, 


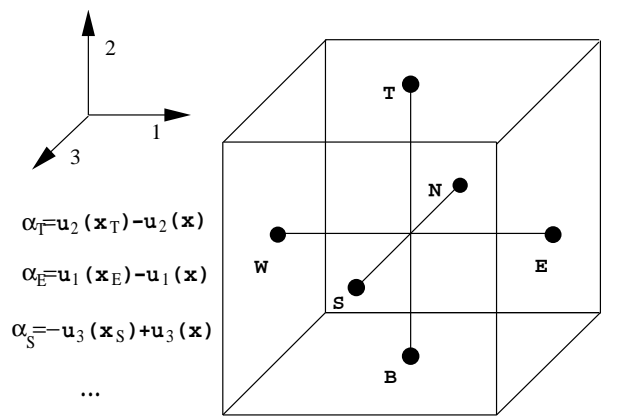

Figure 2: Schéma aux différences correspondant au modèle sous-maille (7) dans le cas d'une grille uniforme. Les poids des différents points de grille sont les parties négatives de $\left(\alpha_{T}\right) / \Delta,\left(\alpha_{E}\right) / \Delta$, etc.

la dissipation ne s'exercera que dans la direction E-W, qui correspond bien aux valeurs propres négatives de $S$.

Les figures 3 et 3 permettent d'évaluer le comportement de ce modèle. La figure 3 montre les spectres d'énergie obtenus dans une expérience de turbulence homogène isotrope classique (dite de Comte-Bellot) pour une simulation directe de reférence utilisant $128^{3}$ points et des simulations de grandes échelles $32^{3}$ utilisant le modèle de Smagorinsky et 2 implémentations différentes (correspondant à 2 formes différentes du cut-off $\zeta$ ) du modèle (7). Les coefficients des différents modèles ont été fixés de manière à reproduire une décroissance correcte d'énergie (figure 3). Le modèle anisotrope apparait clairement moins dissipatif dans les les nombres d'onde proches de l'échelle de coupure, ce qui se traduit par une décroissance de l'enstrophie plus conforme au résultat de la simulation de référence (figure 3). Ceci est confirmé en observant les spectres d'énergie (voir [4]). La figure 3 montre la dissipation sous-maille obtenue dans un calcul de canal turbulent. C'est un cas où le cisaillement est évidemment très important près des parois ce qui rend le modèle de Smagorinsky totalement inopérant, sauf si on y rajoute une loi adhoc contraignant le coefficient à s'annuler à la paroi. Les modèles dynamiques mentionnés dans l'introduction permettent aussi de calculer ce coefficient de manière adaptative en utiisant des hypothèse d'homogénéité de l'écoulement dans les directions parallèles aux parois. D'une manière frappante le modèle anisotrope reproduit un profil de dissipation très similaire ce qui montre qu'il possède de bonnes propriétés asymptotiques près des parois (voir [4] pour des discussions plus détaillées de ce point). Il faut noter que, pour cet écoulement, le modèle anisotrope se comporte de manière satisfaisante, même si l'on conserve sa partie anti-diffusive ("unclipped model" dans la figure 3).

\section{Modèles sous-maille et méthodes Lagrangiennes}

La communauté SGE a commencé - un peu tardivement - à réaliser que les performances des modèles étaient largement tributaires des schémas de discrétisation utilisés. Il est par exemple de plus en plus communément admis que les schémas décentrés, même d'ordre élevés, sont inappro- 

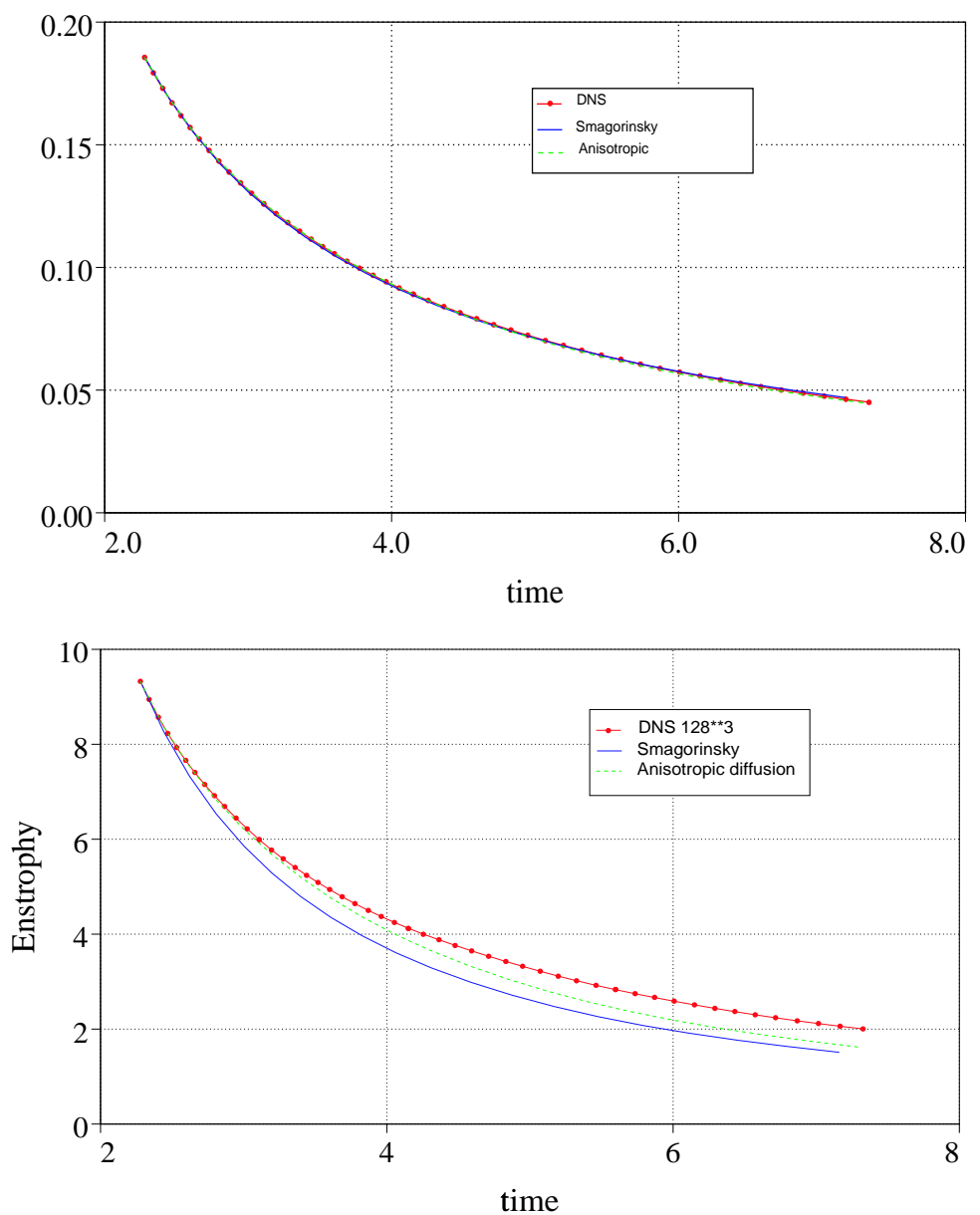

Figure 3: Courbes d'énergie (haut) et d'enstrophie (bas) dans une expérience de turbulence homogène isotrope. Les points concernent les résultats d'une simulation à haute résolution. 

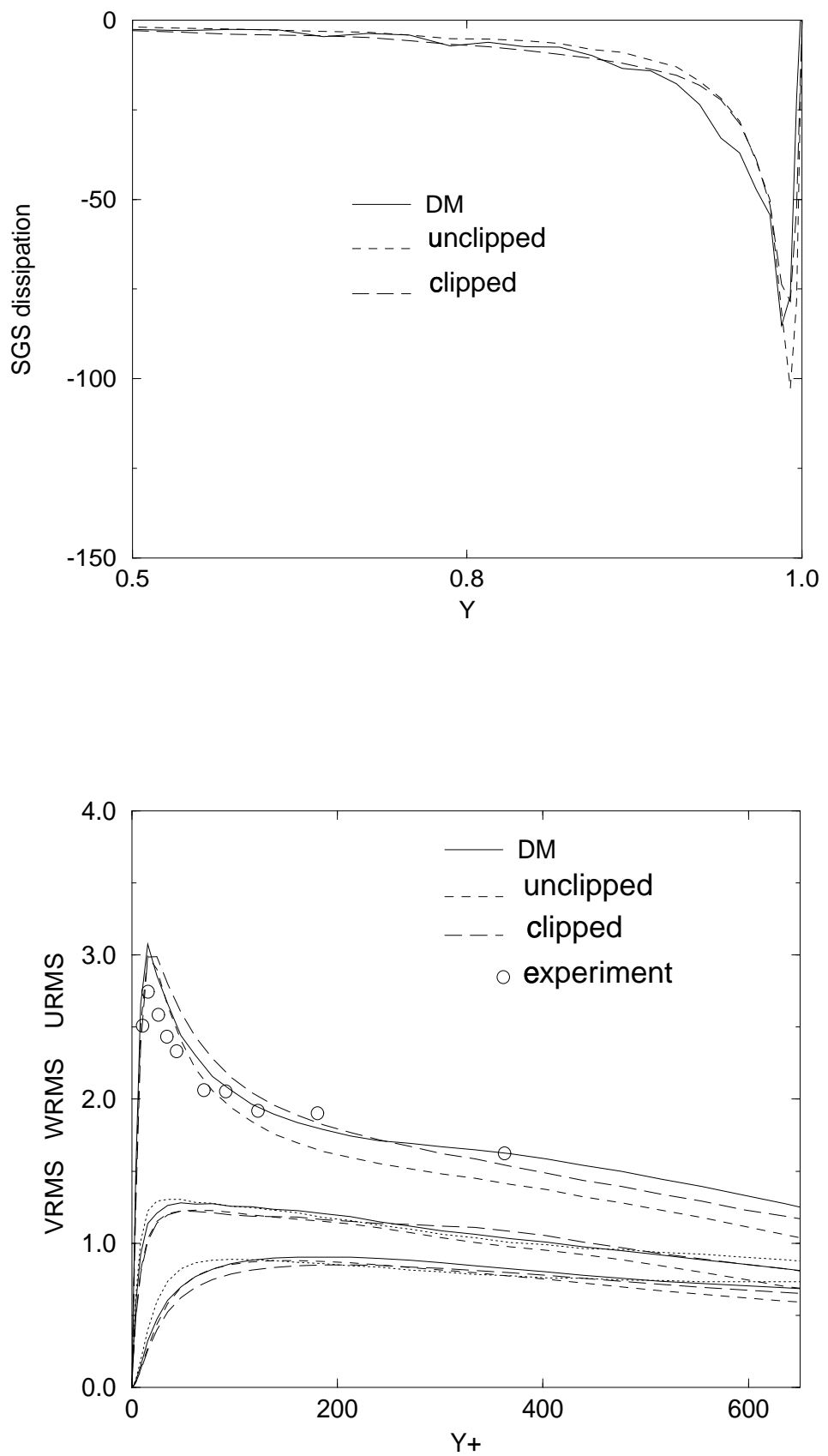

Figure 4: Dissipation sous-maille (haut) et Intensités turbulentes (bas) dans un canal turbulent à Reynolds 22,000. DM: modèle de Smagorinsky dynamique.

ESAIM: Proc., Vol. 11, 2002, 85-95 
priés pour la SGE car ils contiennent une dissipation numérique qui masque les effets des modèles sous-maille. Nous allons voir que les schémas purement Lagrangiens (particulaires) ont par contre des propriétés remarquables qui en font de outils naturels pour la simulation des grandes échelles.

Revenons à la formulation tourbillon des équations, en se concentrant sur le cas d'une viscosité nulle (équations d'Euler). Un schéma particulaire (voir [2] et les références qui y sont indiquées) consiste à adopter une discrétisation de la vorticité sous la forme

$$
\boldsymbol{\omega}(\mathbf{x}, t)=\sum_{p} \boldsymbol{\Gamma}_{p} \delta\left(\mathbf{x}-\mathbf{x}_{p}\right)
$$

Les particules évoluent et modifient leur circulation suivant les trajectoires du système différentiel

$$
\frac{d \mathbf{x}_{p}}{d t}=\overline{\mathbf{u}}\left(\mathbf{x}_{p}, t\right), \frac{d \boldsymbol{\Gamma}_{p}}{d t}=\nabla \overline{\mathbf{u}}\left(\mathbf{x}_{p}, t\right) \boldsymbol{\Gamma}_{p}
$$

où $\overline{\mathbf{u}}$ représente un champ de vitesse régularisé. Ceci revient en fait à résoudre dans un sens faible l'équation de transport

$$
\frac{\partial \omega_{i}}{\partial t}+\operatorname{div} \overline{\mathbf{u}} \omega_{i}-\operatorname{div} \boldsymbol{\omega} \bar{u}_{i}=0
$$

Par conséquent, le champ de vorticité régularisé (c'est à dire celui que est effectivement utilisé pour le calcul des vitesses) satisfait

$$
\frac{\partial \overline{\boldsymbol{\omega}}_{i}}{\partial t}+\operatorname{div} \overline{\overline{\mathbf{u}} \boldsymbol{\omega}_{i}}-\operatorname{div} \overline{\boldsymbol{\omega} \bar{u}_{i}}=0
$$

qui n'est autre qu'une équation d'Euler moyennée. En d'autres termes, le schéma particulaire réalise naturellement, et sans adjonction de modèle, une modélisation sous-maille. On peut retrouver cette propriété remarquable, en réalisant qu'un schéma particulaire, au contraire d'un schéma Eulérien, n'est pas limité à une échelle de coupure prédéterminée, car les particules peuvent se rapprocher, au moins en principe car en pratique on est amené à remailler périodiquement les particules, arbitrairement. Ces schémas contiennent donc par essence un mécanisme de cascade vers les petites échelles. Pour illustrer cette propriété nous montrons dans les figures qui suivent des calculs d'instabilité de Crow avec méthode particulaire et méthode spectrale. Le nombre de Reynolds est de 3500 , et la résolution est de $120^{3}$ pour les 2 schémas, sauf pour un cas où l'on a utilisé $180^{3}$ points. Ces résolutions sont insuffisantes pour que ces simulations puissent être qualifiées de simulations directes. La figure 4 montre les iso-valeurs de vorticité dans le plan de reconnection peu après la reconnection, pour le schéma particulaire, une méthode spectrale déaliasée, et une méthode spectrale utilisant le modèle de Smagorinsky avec la valeur du coefficient habituellement utilisée dans des écoulements homogènes. Il apparait clairement que la méthode spectrale brute produit un bruit numérique, typique d'une accumulation d'énergie dans les petites échelles, qui ne disparait pas totalement avec le modèle de Smagorinsky. La méthode particulaire ne souffre pas de cette difficulté. On pourrait penser que c'est au prix d'une dissipation excessive dans les grandes échelles mais ce n'est pas le cas, comme le montre la courbe d'enstrophie et les spectres dans la direction la plus singulière sur la figure 4 (d'autres résultats peuvent être trouvés dans [3]). 
(a)

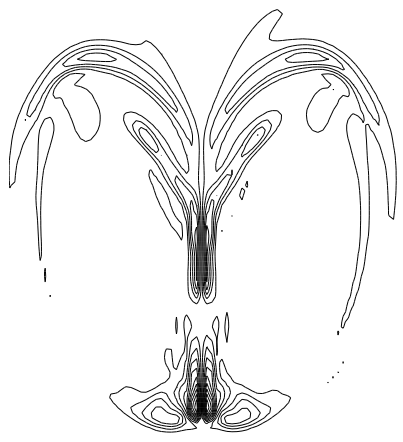

(c)

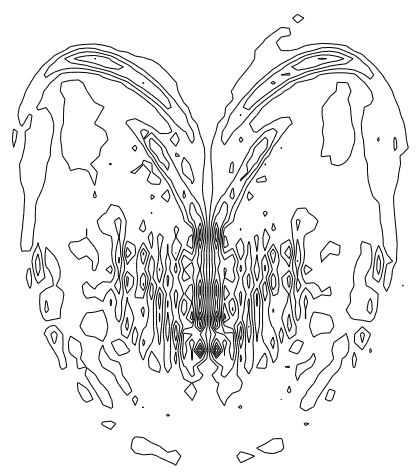

(b)

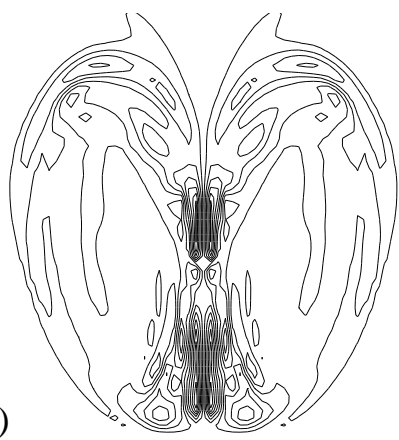

(d)

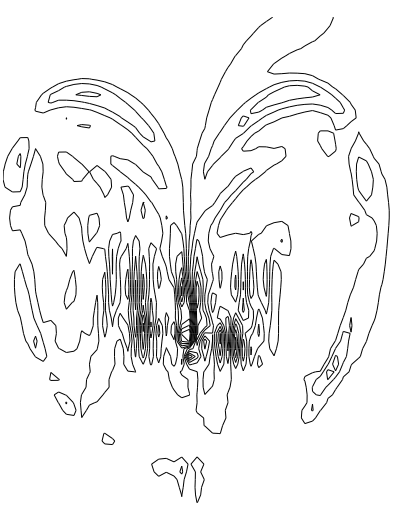

Figure 5: Iso-valeurs de tourbillon dans le plan de reconnection obtenues par: (a) une méthode particulaire à une résolution $180^{3}$; (b): une méthode particulaire à une résolution $120^{3}$; (c): une méthode spectrale dé-aliasée à une résolution $120^{3}$; (d): une méthode spectrale avec modèle de Smagorinsky à une résolution $120^{3}$.
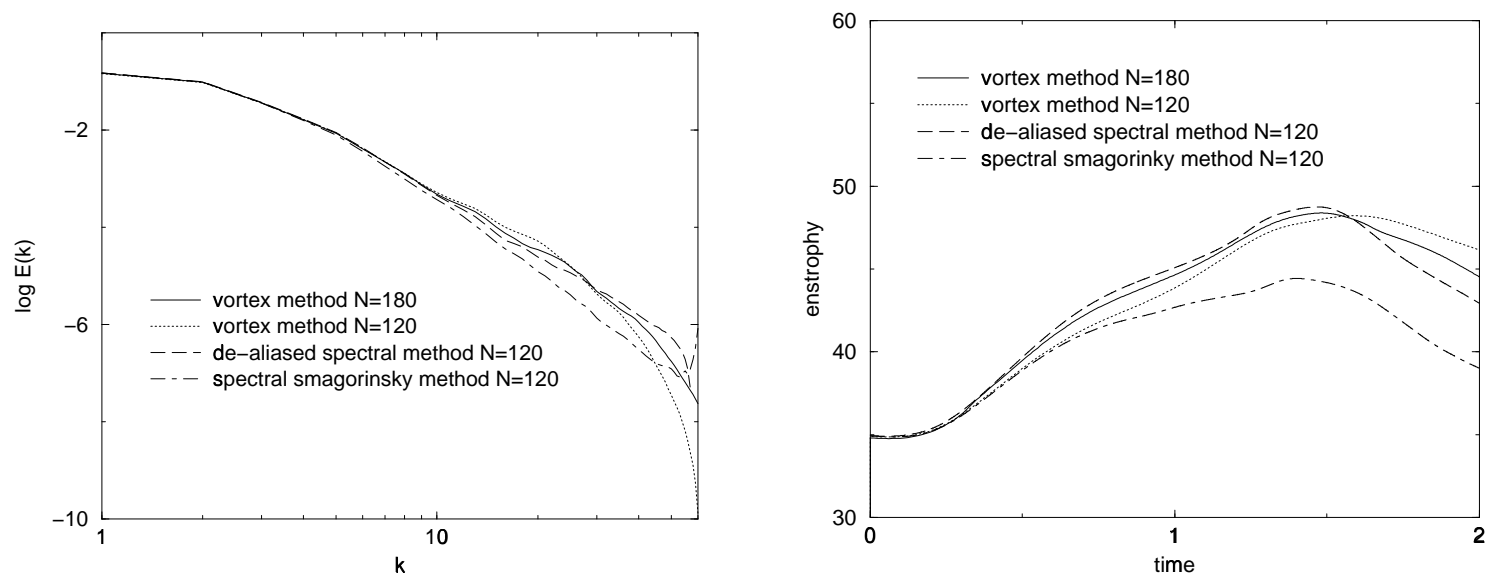

Figure 6: Enstrophie et spectres d'énergie peu après la reconnection dans une instabilité de Crow. 


\section{Conclusion}

On a vu qu'en se posant le problème de l'apparition de singularités dans les équations on aboutit à des modèles de dissipation sous-maille relativement satisfaisants. Ces modèles peuvent s'adapter facilement à des schémas de discrétisation. On peut même montrer (voir [4]) que dans le cas de grille non uniforme ils prennent en compte les échelles éventuellement non isotropes des mailles. Les schémas Lagrangiens quant à eux sont par nature, lorsqu'ils sont sous-résolus, des méthodes de simulation de grandes échelles qui par leur robustesse devraient se révèler des alternatives attractives aux méthodes Eulériennes classiques. 


\section{Bibliographie}

[1] A.J. Chorin, Vorticity and Turbulence, Springer Verlag, 1994

[2] G.-H. Cottet et P. Koumoutsakos, Vortex methods: theory and practice, Cambridge University Press, 2000

[3] G.-H. Cottet, B. Michaux, S. Ossia et G. VanderLinden, A comparison of spectral and vortex methods in three-dimensional incompressible flows, soumis.

[4] G-H. Cottet and A.A Wray, Anisotropic grid-based formulas for subgrid-scale models, Annual Research Briefs, Center for Turbulence Research, 113-122, 1997

[5] M. Germano, U. Piomelli, P. Moin and W.H. Cabot, A dynamic subgrid-scale eddy viscosity model, Phys. Fluids A, 3, 1760-1765, 1991

[6] A. Leonard, Energy cascade in large-eddy simulations of turbulent fluid flows, Adv. Geophys., 18, 237-248, 1974

[7] A. Majda, Vorticity and the mathematical theory of incompressible fluid flow, Comm. Pure Appl. Math., 39, S187-S179, 1986

Georges-Henri Cottet, Delia Jiroveanu \& Bertrand Michaux

LMC-IMAG

Université Joseph Fourier

BP 53

38041 Grenoble Cedex 9, France

mailto:cottet@imag.fr 